Institute of $\mathbf{F}_{\text {ood and }} \mathbf{A}_{\text {gricultural }} \mathbf{S}_{\text {ciences }}$

\title{
Red Wax Scale, Ceroplastes rubens Maskell (Insecta: Homoptera: Coccidae) ${ }^{1}$
}

G.W. Dekle ${ }^{2}$

\section{Introduction}

Red wax scale, Ceroplastes rubens Maskell, was first found in Florida at an Orange County nursery on the foliage and stem of greenhouse-grown Aglaonema pictum var. tricolor and A. oblongifolium 'Curtisii' in November 1955. This collection was a new Florida and continental United States record of an introduced plant pest. Introduced foliage plants apparently were responsible for the establishment of this insect in Florida.

\section{Distribution}

Antigua, Australia, Bermuda, Caroline Is., China, Cook Is., Cuba, Dominican Republic, Fiji, France, Guam, Hawaii, Honduras, India, Indonesia, Italy, Japan, Kenya, Loyalty Islands, Malaya, Mariana Islands, Mexico, New Caledonia, New Zealand, Philippines, Samoa, Seychelles, Solomon Is., South Africa, Sri Lanka, Tahiti, Taiwan, Vanuatu and Zanzibar.

\section{Description}

The waxy cover of the adult female is pinkish to red, convex, longer than wide and with two conspicuous pairs of white bands that extend dorsally from the spiracular area. Size: 3.5 to $4.5 \mathrm{~mm}$. The nymphs are pinkish in color.

\section{Hosts}

Acanthacae, Acer sp. (maple), Acrostichum sp. (fern), Aglaonema commutatum Schott, A. modestum Schott, A. nitidum (jack) Kunth 'Curtisii', A. pictum 'Tricolor', A. commutatum Schott 'Pseudobracteatum', A. roebelinii (Lind.) Gentil., A. treubii Engl., Allamanda cathartica L. 'Schotti', Alpinia purpurata K. Schum., Alyxia olivaeformis Gaudich., Anthurium sp., Antidesma sp. (China laurel), Artemisia vulgaris L. (mugwort), Ardisia japonica Blume, Asplenium nidus L. (bird nest fern), Aucuba sp. (golddust plant), Berberis sp. (barberry), Brassaia actinophylla Endl., (schefflera), Buxus sp. (boxwood), B. microphylla Sieb., Calophyllum tomentosum Wight. (beautyleaf), Camellia japonica L., C. japonica L., ssp. rusticana (Honda) Kitamura,

1. This document is EENY-237 (originally published as DPI Entomology Circular 115), one of a series of Featured Creatures from the Entomology and Nematology Department, Florida Cooperative Extension Service, Institute of Food and Agricultural Sciences, University of Florida. Published: September 2001. This document is also available on Featured Creatures Website at http://creatures.ifas.ufl.edu. Please visit the EDIS Website at http://edis.ifas.ufl.edu. Additional information on these organisms, including many color photographs, is available at the Entomology and Nematology Department website at http://entnemdept.ifas.ufl.edu/.

2. G.W. Dekle, Florida Department of Agriculture and Consumer Services, Division of Plant Industry, Gainesville, FL.

The Institute of Food and Agricultural Sciences is an equal opportunity/affirmative action employer authorized to provide research, educational information and other services only to individuals and institutions that function without regard to race, color, sex, age, handicap, or national origin. For information on obtaining other extension publications, contact your county Cooperative Extension Service office. Florida Cooperative Extension Service/Institute of Food and Agricultural Sciences/University of Florida/Christine Taylor Waddill, Dean. 


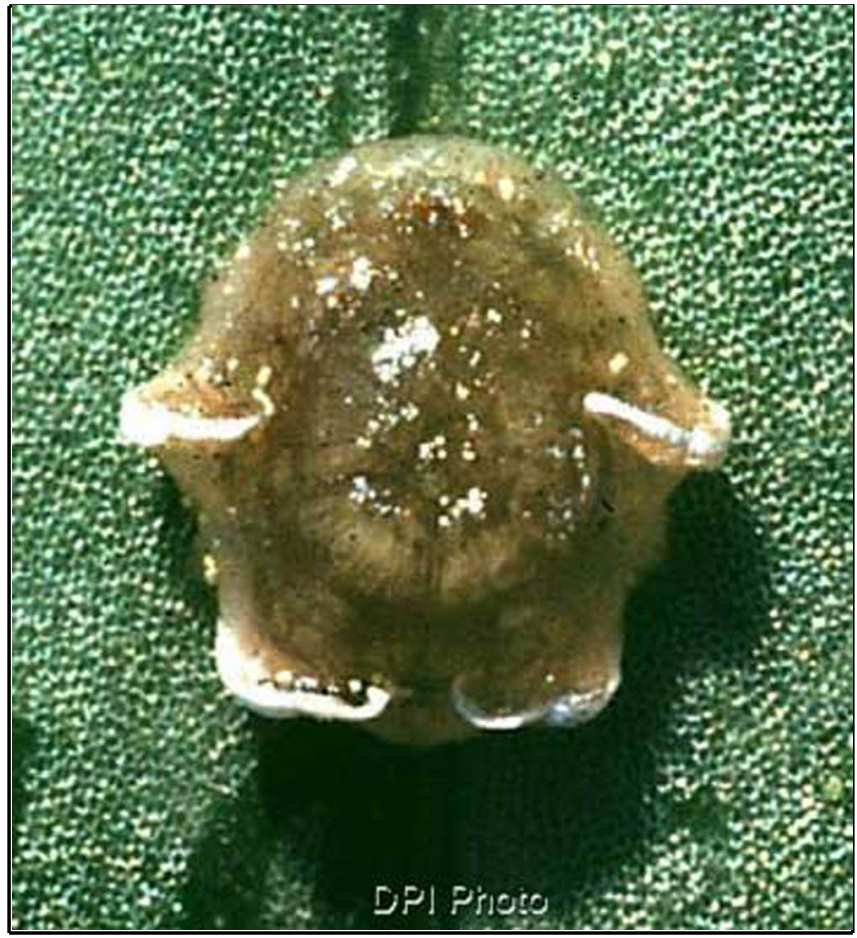

Figure 1. Adult female red wax scale, Ceroplastes rubens Maskell, showing the conspicuous pairs of white bands. Credits: Division of Plant Industry

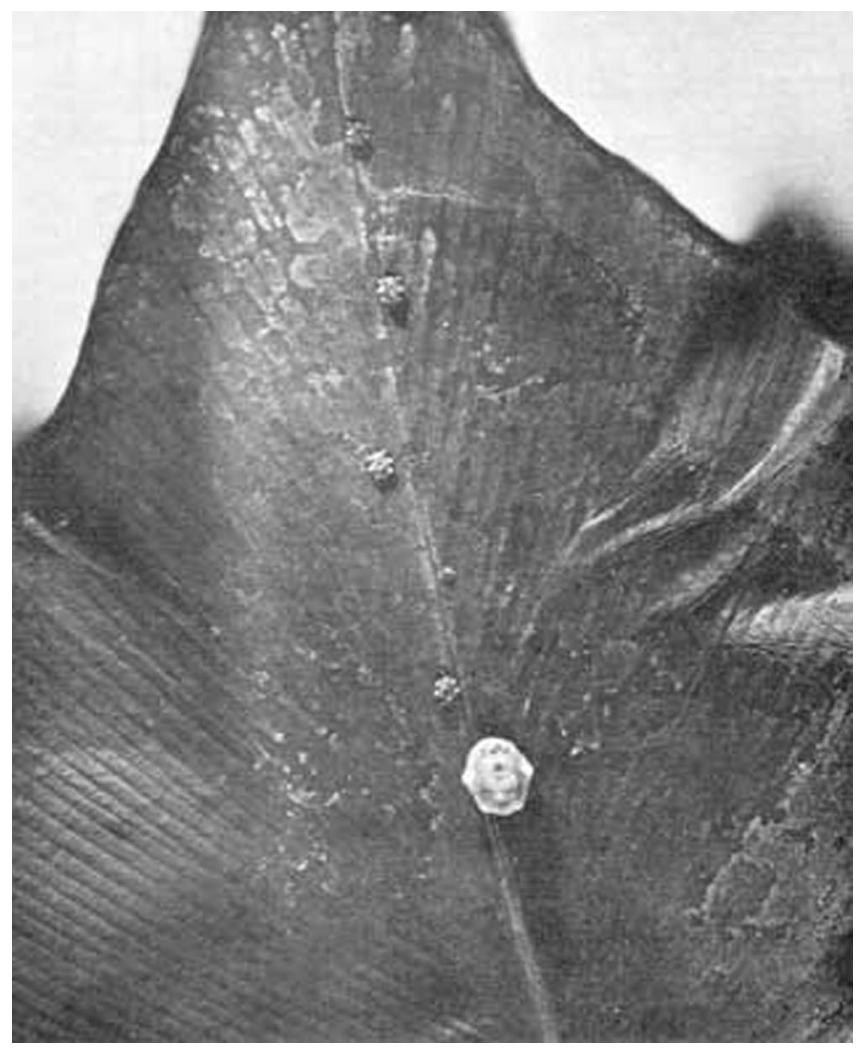

Figure 2. Adult female and nymphs of the red wax scale, Ceroplastes rubens Maskell. Credits: Division of Plant Industry
C. sinensis (L.) Kuntze (tea), Capsicum annum L. (pepper), Celosia argentea L., var. cristata (L.) Kuntze, Chaenomeles japonica (Thunb.) Lindl. (flowering quince), Chrysanthemum sinense Sabine, Cibotium sp. (fern tree), Cinnamomum pedunculatum Presl. (cinnamon), Citrus reticulata Blanco (mandarin orange), C. sinensis (L.) Osbeck (sweet orange), Cleyera japonica Thunb., Cocos nucifera L. (coconut), Coffea sp. (coffee), Cotoneaster sp. (ornamental), Crassula rosularis Haw. (succulent), Crataegus sp. (haw, hawthorn, mayhaw), Cycas revoluta Thunb. (Sago palm), Cydonia oblonga Mill. (quince), Cytisus scoparius Link (Scotch broom), Daphne odora Thunb., Dieffenbachia sp. (ornamental), Dioclea violacea Mart. ex Benth., Diospyros kaki L.f. (Japanese persimmon), Dizygotheca elegantissima Vig. \& Guill. (false aralia), Duranta repens L. (skyflower), Elaecarpus bifidus Hook. \& Arn., Elaphoglossum reticulatus Gaud. (fern), Epidendrum sp. (orchid), Eriobotrya japonica Lindl. (loquat), Eucalyptus globulus Labill. (Tasmanian bluegum), Eugenia smithii Poir., Euonymus europaens L. (shrub), E. japonicus Thunb. (Japanese evergreen), E. alata Maxim., Eurya emarginata (Thunb.) Makino, Fatsia japonica Decne. \& Planch. (aralia), Ficus carica L. (fig), F. faveolata Wall., Garcinia spicata Hook.f., Gardenia jasminoides Ellis (gardenia, Cape jasmine), Grammatophyllum sp. (orchid), Hedera helix L. (English ivy), Ilex serrata Thunb. (holly), Illicium anisatum L. (anise tree), Iresine sp. (bloodleaf), Ixora sp. (evergreen shrub), Kadsura japonica (Thunb.) Dunal. (Katsura tree), Laurus nobilis L. (laurel), Ligustrum japonicum Thunb. (privet), Lindera citridora Hemsl. (spice bush), Litchi chinensis Sonn. (lychee, leechee), Litsea glauca Sieb., Lycium chinense Mill. (Chinese matrimony vine), Machilus thunbergii Sieb., Magnolia salicifolia (bay), $M$. obovata Thunb. (bay), Malus sp. (apple), Mangifera indica L. (mango), Medinilla sp., Melia azedarach L. (chinaberry, china tree), Metrosideros villosa Sm., Monstera deliciosa Liebm., Morus alba L. (white mulberry), Myrsine sp., Nandina domestica Thunb., Nerium oleander L. (oleander), Paltonium lanceolatum Presl. (ribbon fern), Peperomia sp. (peperomia), Persea americana Mill. (avocado), Philodendron giganteum Schott, Photina glabra Maxim., Pilea L., Pinus densiflora Sieb. \& Zucc., P. 
parviflora Sieb. \& Zucc. (Japanese white pine), Pittosporum tobira (Thunb.) Ait. (pittosporum), Podocarpus nagi (Thunb.) Makino (podocarpus), Polypodium sp. (fern), Poncirus trifoliata (L.) Raf. (trifoliate orange), Prunus domestica L. (peach or prune), P. mume Sieb. \& Zucc., Psychotria sp. (wild coffee), Pteris sp. (brake fern), Pyrus pyrifolia (Burm.f.) Nakai (sand pear), Rhododendron indicum Sweet (azalea), Rhus succedanea L. (wax-tree), Rosa sp. (rose), Schima sp., Spartium junceum L. (Spanish broom), Spirea thunbergii Sieb. (spirea, bridalwreath), Stanhopea sp., Straussia sp., Strobilanthes japonicus Miq. (lavenderbell), Symplocos japonica DC., Todea barbara (L.) Moore, Tamarix chinensis Lour. (salt cedar, tamarix), Wisteria sp. (wisteria), Zantedsechia sp. (calla).

\section{Economic Importance}

This scale has been reported to be an economic pest on citrus.

\section{Management}

See the Insect Management Guide for Woody Ornamentals.

\section{Selected References}

Borchsenius, N.S. 1957. Soft Scales of the USSR (Coccidea). New Series, No. 66. National Academy of Sciences of the USSR. Moscow and Leningrad. $494 \mathrm{p}$.

Commonwealth Institute of Entomology. 1960. Distribution Maps of Pests. (Series A Agricultural), Map No. 118.

Dekle, G.W. 1957. Twenty-First Biennial Report, State Plant Board of Florida, now Div. Plant Ind., Florida Dept. Agr. \& Consumer Serv., Vol. II, Bull. 11-A: 73-74.

Green, E.E. 1909. The Coccidae of Ceylon. Part IV. Dulae \& Co., London. 280-281, 15 figs.

Zimmerman, E.C. 1948. Insects of Hawaii, Volume 5, Homoptera: Sternorhyncha, 343-347. 